\title{
$\begin{array}{ll}\text { Research Square } & \text { Preprints are preliminary reports that have not undergone peer review. } \\ \text { They should not be considered conclusive, used to inform clinical practice, }\end{array}$ or referenced by the media as validated information.
}

\section{Coronary Tortuosity Overestimate The FFR Measurement In Stenotic Coronary Artery Model}

Jiatong Liu

General Hospital of Northern Theatre command

Libo Zhang

General Hospital of Northern Theatre command

Hongzeng Xu ( $\sim$ hongzengxu@qq.com)

Liaoning Provincial Key Laboratory of Cardiovascular Imaging Medicine, General Hospital of Northern Theater Command, 110016, China. https://orcid.org/0000-0002-1866-3190

Research article

Keywords: Coronary artery disease, Hemodynamics, FFR

Posted Date: January 16th, 2021

DOl: https://doi.org/10.21203/rs.3.rs-144020/v1

License: (c) (i) This work is licensed under a Creative Commons Attribution 4.0 International License. Read Full License 


\title{
Coronary Tortuosity overestimate the FFR measurement in stenotic coronary artery model
}

Jiatong Liu ${ }^{\mathrm{a}}$, Libo Zhang ${ }^{\mathrm{b}}$, Hongzeng $\mathrm{Xu}^{\mathrm{b}^{*}}$

\author{
a. Department of Anesthesiology, General Hospital of Northern Theater Command, Shenyang 110016, China. \\ b. Liaoning Provincial Key Laboratory of Cardiovascular Imaging Medicine, General Hospital of Northern Theater \\ Command, 110016, China.
}

*Corresponding author: Hongzeng Xu, Liaoning Provincial Key Laboratory of Cardiovascular Imaging, General Hospital of Northern Theater Command, No.83 wenhua Road, Shenyang 110016, China. Tel: +86-24-28856123, Email: hongzengxu@qq.com

Running Title: Coronary Tortuosity overestimate FFR 


\section{Abstract:}

Background: The object of the study is to investigate the effect of coronary tortuosity (CT) on fractional flow reserve (FFR) in stenotic coronary artery.

Methods: A three dimensional computational model of simulation of blood flow in stenotic coronary artery with multi-bend CT was constructed with Fluent 16.0 software. Blood was simulated as non-Newtonian fluid with the Carreau model. The simulation of blood flow in coronary artery stenotic model was used by the finite element methods with the condition of CT and no coronary tortuosity (NCT). Coronary artery hemodynamic parameters such as pressure, velocity and physiological diagnostic parameter fractional flow reserve (FFR) were studied in the model with the coronary tortuosity condition.

Results: The results showed that the downstream CT impedance condition has significant impacts on numerical simulation. The pressure profile of pre-stenotic is almost identical in the two models. However the pressure in the pre-stenotic and post-stenotic artery domain is much higher in the CT model. The pressure fluctuation range in $\mathrm{CT}$ model was much higher than that in the NCT model. In the coronary artey model with $75 \%$ stenosis for the CT condition, the FFR was 0.823 while the FFR was 0.767 in the same model with NCT condition.

Conclusions: This study provides evidence that FFR value was increased in coronary stenotic artery with the presence of CT. Therefore, it should be taking into account the influence of CT load effect in FFR measurement procedure, otherwise the CAD risk will be underestimated.

Keywords: Coronary artery disease, Hemodynamics, FFR 


\section{Background}

Arteries are normally straight to transport blood and oxygen to distal tissues efficiently. However, arteries may become tortuosity as a many result of arterial remodeling factors such as left ventricular hypertrophy or due to elongation and dilation of the arteries associated with hypertension, smoking and aging [1]. Tortuosity was identified by $\geq 3$ bends segments (defined as $\geq 45^{\circ}$ change in vessel main direction) along main trunk of one artery. Tortuous coronary arteries (Fig.1.A) and non-tortuous coronary arteries (Fig.1.B) are two geometric patterns of coronary arteries commonly observed in clinical coronary angiography. Although CT can influence the hemodynamics of coronary arteries [2], the relationship between CT and fractional flow reserve (FFR) has not been investigated.

Regulation of coronary blood flow is quite complex, the coronary artery hemodynamics are affected by the multi-factor such as epicardial stenotic coronary artery obstruction, CT and microcirculation load. Cardiologists often encounter coronary tortuosity. However, the complex interrelationship between the tortuous coronary arteries and the stenotic epicardial coronary arteries contributing to the coronary artery hemodyanamics remains poorly understood. Whether CT leads to an apparent FFR change to the stenotic coronary artery model is still unknown.

Computational fluid dynamics (CFD) simulation has been widely used to study the hemodynamic parameters in heart due to the limitation of in vivo measurements [3]. Hemodynamics is a key factor in the onset of vascular wall pathologies. Functional evaluation of coronary artery stenosis severity is important in clinical practice [4]. FFR is currently used as a gold standard for the assessment of functional significance of stenosis severity and is applied for guiding cardiovascular intervention [5]. Recently, the CFD approach has been used to determine the FFR from the clinical medical image or numerical simulation. However, there are lack of numerical simulation studies to determine FFR and hemodynamic changes due to coronary artery tortuosity (CAT) conditions. We hypothesize that hemodynamic changes caused by CT may partly obscure FFR measurements. The purpose of this study is to investigate the CT hemodynamic effect on FFR in stenotic coronary 
artery.

\section{Methods}

\section{Geometry Model}

As shown in Fig.2, the coronary artery model was considered as a symmetric stenotic geometry with the condition of CT and no coronary tortuosity (NCT) condition. Tortuous coronary arteries segments were designed as 4 bends (with $>45^{\circ}$ change in vessel direction). Stenotic regions consist of throat, converging and diverging sections. Moreover, proximal and distal radii are assumed to be identical $\left(\mathrm{r}_{\mathrm{p}}\right.$ $\left.=r_{d}=1.4 \mathrm{~mm}\right)$. Artery area stenosis (AS) percentage was $75 \%$, which was defined as:

$$
\frac{D_{1}^{2}-D_{s}^{2}}{D_{1}^{2}} \times 100 \%
$$

The 3D geometry was created with SolidWorks with shape and dimensions as close as possible to the human artery.

\section{Computational blood flow model}

The simulation was performed under laminar flow conditions. The flow of blood in the coronary artery was assumed as incompressible and governed by the Navier-Stokes equations

$$
\rho\left(\frac{\partial_{u}}{\partial_{t}}+u \cdot \nabla u\right)=-\nabla p+\nabla \cdot T
$$

and the continuity equation for incompressible flow was based on the mass conservation principle and the general form was written as

$$
\nabla \cdot u=0
$$

Where $\mathrm{t}$ indicated the time, $u$ the three dimensional velocity vector, $p$ the pressure $\rho$ the blood density and $T$ the stress tensor respectively. The blood was set with a density of $1050 \mathrm{~kg} / \mathrm{m}^{3}$.

As is well known, the viscoelasticity and shear thinning of the blood are closely relevant to its microscopic features, such as deformation, aggregation and alignment 
of the red blood cells and shouldn't be neglected in the blood blood flow simulation [6]. In this study, the blood flow was modeled to be homogenous, incompressible and non-Newtonian, and the Carreau blood viscosity model was used which is governed by the equation given as

$$
\mu=\mu_{\infty}+\left(\mu_{0}-\mu_{\infty}\right)\left[1+(\lambda \gamma)^{2}\right]^{(n-1) / 2}
$$

where $\mu$ is the dynamic viscosity, $\mu_{0}$ and $\mu_{\infty}$ are the viscosity as the shear rate goes to infinity and zero, $\lambda$ is the time constant, $\gamma$ is the shear rate and $n$ is the power-law index. All values were taken from the literature, $\mu_{0}=0.0560 \mathrm{~Pa} \cdot \mathrm{S}, \mu_{\infty}=0.00345$ $\mathrm{Pa} \cdot \mathrm{S}, \lambda=3.313 \mathrm{~s}$ and $\mathrm{n}=0.3568$.

\section{Microcirculation domain}

In this study, the outlet impedance was set as seepage boundary condition which is provided by the flow in the microcirculation porous zone [7]. Microcirculation is considered as a porous medium which is the important part of the whole circulation structure, A complete hemodynamic model requires consideration of the microcirculation load effect. The porosity of the microcirculation was set to $\phi=0.5$. We adopted the following empirical equation to compute the microcirculation zone permeability, which is determined by the diameter and porosity:

$$
\mathrm{k}=\frac{d^{2} \phi^{3}}{180(1-\phi)^{2}}
$$

where $\mathrm{d}$ is the diameter of the microvessel, and we set $\mathrm{d}=100 \mu \mathrm{m}$ following the literature [8]. The blood flow governing equations in the microcirculation zone are determined as follows:

$$
\begin{aligned}
& \rho\left(\frac{\partial_{(\varphi u)}}{\partial_{t}}+u \cdot \nabla(\varphi u)\right)=-\nabla(\varphi p)+\nabla \cdot(\varphi T)-\frac{\varphi^{2} \eta}{k} u \\
& \nabla \cdot u=0
\end{aligned}
$$




\section{Boundary conditions and microcirculation domain}

All calculation examples selected velocity inlet condition and free outlet, which was used for simulation the process of artery blood flow impulse. A transient time-dependent velocity profile of a typical coronary artery was applied at the inlet. The maximum velocity was $0.46 \mathrm{~m} / \mathrm{s}$, the average velocity was $0.28 \mathrm{~m} / \mathrm{s}$ and the minimum velocity was $0.13 \mathrm{~m} / \mathrm{s}$. Accordingly, in the example of normal physiology, the heart rate was $75 / \mathrm{min}$ and the period of the flow waveform is $0.8 \mathrm{~s}$. Both CT and NCT cases were solved with the same inlet boundary conditions. The microcirculation zone is coupled at the outlet.We assume that the vessel walls are rigid and all velocity components were set to zero at the wall according to no-slip condition. Coronary artery hemodynamic parameters such as pressure, velocity and physiological diagnostic parameter FFR were investigated in the model.

\section{Numerical methodology}

The computational domains were meshed with tetrahedral and hexahedral elements using ANSYS Meshing (Ansys Inc., Canonsburg, USA). The total number of elements vary from 60,000 to 78,000 for both CT and NCT models. Computational simulations were performed with commercial CFD software Fluent 16.0 using the numerical scheme of finite-volume analysis. The transient flow analysis was run for 114 time steps (0.007 s per time step) representing pulsatile flow with each time step converging to a residual target of $1 \times 10^{-5}$.

\section{FFR calculation}

FFR is the most well-studied physiological parameter to guide coronary revascularization decision in contemporary clinical practice [9]. FFR is defined as the ratio of distal coronary pressure to proximal (=aortic) pressure determined during pharmacologically-induced hyperemia

$$
\mathrm{FF} R=\frac{\tilde{\mathrm{P}}_{d}-\mathrm{P}_{v}}{\tilde{\mathrm{P}}_{a}-\mathrm{P}_{v}}
$$

Where $\tilde{\mathrm{P}}_{d}$ is the time averaged distal stenotic pressure measured at the end of flow 
reversal occurring and before the $\mathrm{CT}$ bending segments, $\tilde{\mathrm{P}}_{a}$ is defined as the time averaged aortic pressure, and $\mathrm{P}_{\mathrm{v}}$ is the venous pressure which is assumed to be 15 $\mathrm{mmHg}$.

\section{Results}

In the model with CT and NCT condition, the present study showed the different hemodynamic parameters. Fig.3 depict the pressure distributions before and after stenosis of the artery zone. In the two models, the pressure profiles of the pre-stenotic and post-stenotic domain are almost identical. However, the average of pre-stenotic pressure $\left(\tilde{\mathrm{P}}_{a}=3707 \mathrm{~Pa}\right)$ and post-stenotic pressure $\left(\tilde{\mathrm{P}}_{d}=3051 \mathrm{~Pa}\right)$ in the CT model

were higher than those $\left(\tilde{\mathrm{P}}_{a}=2890 \mathrm{~Pa}, \tilde{\mathrm{P}}_{d}=2217 \mathrm{~Pa}\right)$ in the NCT model. The absolute values of the pressure drops across the stenosis were similar in the two models. However, the pressure fluctuation range in CT model was much higher than that in NCT model.

After obtained the averaged pressure distal $\left(\tilde{\mathrm{P}}_{d}\right)$ and proximal $\left(\tilde{\mathrm{P}}_{a}\right)$ to stenosis from our computational study, FFR $\left(=\tilde{\mathrm{P}}_{d} / \tilde{\mathrm{P}}_{a}\right)$ was calculated. As shown in Table 1, a large pressure drop occurs across the stenosis. In the coronary artey model with $75 \%$ stenosis for the CT condition, the FFR was 0.8228 while the FFR was 0.7669 in the same model with NCT condition. The results showed that with the presence of CT resistance, blood flow through the fixed stenosis will decrease, and FFR will increase.

So according to the recent clinical guideline, with the criterion of FFR $=0.80$, the coronary artery model with $75 \%$ stenosis and NCT condition will be judged culprit vessel and require revascularization to prevent adverse events, however the same stenotic vessel with CT condition will be judged nonsignificant. So the FFR value was overestimated in the CT upload condition.

\section{Discussion}

This is the first study to investigate the impact of CT on FFR quantitatively. Little attention has been paid on the impact of coronary tortuosity on FFR before. In the present study, we constructed the artery stenosis model with CT and NCT 
conditions and we study the impact of CT on the pressure and flow field and hence the diagnosis parameter FFR of the artery stenosis model. It can be demonstrated that the downstream CT impedance of artery plays a important role when comparing with the local resistance created by the stenosis district even in the severe case.

Although severe coronary tortuosity (SCT) is relatively common in clinical coronary angiograms, there is much debate on the significance of this anatomical variation and whether CT could reduce the rate of coronary artery disease [1]. Population studies have shown that CT decrease the risk of coronary artery disease [10]. Recent Li's study showed that CT is negatively correlated with coronary atherosclerosis [11]. However, some study showed that CT would alter the local wall shear stress and may become a risk factor for CAD [12, 13]. And Zegers's study showed that atherosclerosis is more common in patients with coronary artery tortuosity [14]. Numerical study suggested that SCT could lead to atherosclerosis [15, 16], and the presence of CT is an hemodynamic factor for the development of atherosclerosis. It has been suggested that CT causes reduction in coronary artery pressure distal to the coronary tortuous segment and alteration in blood flow and thus lead to chest pain. And there is a significant association between SCT and reduced coronary flow rate.

FFR is a well-validated clinical parameter derived from the measurement of coronary pressures and has greatly changed revascularization decision in clinical practice worldwide [17]. And now FFR is regarded as a gold-standard test for myocardial ischemia. FFR is impacted by multi-factor such as artery stenosis and microcirculation impedance [7]. The impact of CT on FFR was not studied previously. Our study showed that FFR was higher than the NCT model with the presence of severe CT. A full understanding of hemodynamic changes caused by the CT is meaningful for cardiovascular researches. CT has important impact on coronary hemodynamics. A 2-D computational experimental studies have shown that CT decrease the blood pressure along the coronary artery [18]. It's believed that the CT has a great influence on the bloodstream, and has important influence on various phenomena in the flow field. The tortuous segment of the coronary artery is a resistant 
factor to coronary atherosclerotic stenosis and reduce the myocardial perfusion [16]. Usually CT was neglected in the procedure of FFR measurement. the tortuous segment of the coronary artery is resistant to coronary atherosclerotic stenosis, and myocardial ischaemia may be caused by irregularities of both epicardial arteries and downstream CAT.

In clinical, coronary tortuosity is common, but its impacts and mechanisms are not well studied. SCT may lead to angina, positive stress test, or other manifestations of myocardial ischemia. It was also shown that SCT is associated with increased Thrombolysis in Myocardial Infarction count, further emphasizing the slower flow rate in comparison to non-SCT patients. Moreover, in this study, it can be demonstrated that the downstream CT impedance of artery plays a dominant role in the evaluation hemodynamic parameter. And such hemodynamic alterations with altered CT resistance could partly obscure FFR measurements. In FFR measurement procedure, the vasodilation mediator is used to abolish all vasoconstrictor tone. However, the tortuous coronary artery impedance couldn't be abolished by the coronary vasodilation drugs. And the CT will cause functional stenosis. A specific constant pressure profile is usually imposed at the outlet in traditional computational hemodynamics simulation. However, free outlet and constant load outlet do not meet the physiological requirements, and microcirculation load outlet condition can better simulate the physiology situation. In our another study, the porous media of the microcirculation is shown to play a important role in the FFR calculation [7].

In this study, the pressure fluctuation range in the CT model was larger than in the NCT model. Our results show that the tortuous coronary arteries seems to have an essential role in regulating blood pressure. And other study showed tortuous coronary arteries may represent larger resistance to blood flow up to about $90 \%$ during exercise conditions that may lead to failure of coronary autoregulation [2]. CT is considered a predictor of abnormal coronary hemodynamics leading to reduced perfusion. Hemodynamic factors are important for the remodeling of the vascular structure, and it's meaningful for cardiovascular clinical researches to understand the hemodynamic changes caused by the CT. Therefore, in hemodynamic numerical simulation study, 
the influence of CT must be considered.

\section{Study limitations and future directions}

The present study has some limitations because of simplifying the simulation. First, an ideal cylinder model was constructed rather than the patient-specific case. Clinically, the artery of the patient may be bifurcated and be deformed due to the myocardial tension, so the haemodynamic research of that may show different outcomes. In addition, there is a fluid-structure interaction (FSI) and a seepage boundary condition between blood and arterial wall, tissues and organs surrounding the coronary artery during the modeling and simulation. This study didn't included the FSI effect. Thus in future work, a more severe case may be constructed included FSI study in association with the model using a seepage boundary condition. Thirdly, factors that influence the hemodynamic parameters such as heart motion, lesion curvature and wall roughness were not included in this study. Using the data from the clinical and further experimental study would validate the computational results in the future as well.

\section{Conclusions}

In conclusion, FFR value was increased with the presence of CT boundary condition in coronary stenotic artery model. Therefore, it should be taking into account the influence of CT load effect in FFR measurement procedure, otherwise the CAD risk will be underestimated. The clinical implication, long-term prognosis and exact pathogenesis of CAT are needed to further study.

\section{Abbreviations}

CT: Coronary tortuosity; FFR: fractional flow reserve; NCT: no coronary tortuosity; SCT: severe coronary tortuosity; CAD: coronary artery disease.

\section{Acknowledgements}

The authors would like to thank Dr Chunlin Zhao for his excellent help with the construction vascular geometrical model.

\section{Sources of funding}

None. 
The datasets used and analysed during the current study are available from the corresponding author on reasonable request

\section{Authors' contributions}

HZX and JTL participated in the design, coordination, and interpretation of this study.

HZX, JTL and LBZ did the CFD numerical modeling experiments. JTL and LBZ wrote this manuscript. All authors read and approved the final manuscript.

Ethics approval and consent to participate

The study was an numerical simulation study and needn't ethics approval.

\section{Consent for publication}

Not applicable.

\section{Competing interests}

The authors declare that they have no competing interests. 


\section{Reference}

1. Khosravani-Rudpishi M, Joharimoghadam A, Rayzan E: The significant coronary tortuosity and atherosclerotic coronary artery disease; What is the relation? J Cardiovasc Thorac Res 2018, 10(4):209-213.

2. Buradi A, Mahalingam A: Impact of coronary tortuosity on the artery hemodynamics. Biocybernetics and Biomedical Engineering 2020, 40(1):126-147.

3. Vorobtsova N, Chiastra C, Stremler MA, Sane DC, Migliavacca F, Vlachos P: Effects of Vessel Tortuosity on Coronary Hemodynamics: An Idealized and Patient-Specific Computational Study. Annals of biomedical engineering 2016, 44(7):2228-2239.

4. Garcia D, Harbaoui B, van de Hoef TP, Meuwissen M, Nijjer SS, Echavarria-Pinto M, Davies JE, Piek JJ, Lantelme P: Relationship between FFR, CFR and coronary microvascular resistance - Practical implications for FFR-guided percutaneous coronary intervention. PloS one 2019, 14(1):e0208612.

5. Parikh RV, Liu G, Plomondon ME, Sehested TSG, Hlatky MA, Waldo SW, Fearon WF: Utilization and Outcomes of Measuring Fractional Flow Reserve in Patients With Stable Ischemic Heart Disease. Journal of the American College of Cardiology 2020, 75(4):409-419.

6. Elhanafy A, Guaily A, Elsaid A: Numerical simulation of blood flow in abdominal aortic aneurysms: Effects of blood shear-thinning and viscoelastic properties. Mathematics and Computers in Simulation 2019, 160:55-71.

7. $\mathrm{Xu} \mathrm{H}$, Liu J, Zhou D, Jin Y: Influence of microcirculation load on FFR in coronary artery stenosis model. BMC Cardiovascular Disorders 2020, 20(1):144.

8. Coccarelli A, Prakash A, Nithiarasu P: A novel porous media-based approach to outflow boundary resistances of 1D arterial blood flow models. Biomechanics and Modeling in Mechanobiology 2019, 18(4):939-951.

9. De Bruyne B, Fearon WF, Pijls NH, Barbato E, Tonino P, Piroth Z, Jagic N, Mobius-Winckler S, Rioufol G, Witt N et al: Fractional flow reserve-guided PCI for stable coronary artery disease. The New England journal of medicine 2014, 371(13):1208-1217. 
10. Li Y, Shen C, Ji Y, Feng Y, Ma G, Liu N: Clinical implication of coronary tortuosity in patients with coronary artery disease. PloS one 2011, 6(8):e24232.

11. Li Y, Feng Y, Ma G, Shen C, Liu N: Coronary tortuosity is negatively correlated with coronary atherosclerosis. Journal of International Medical Research 2018, 46(12):5205-5209.

12. Han HC: Twisted blood vessels: symptoms, etiology and biomechanical mechanisms. Journal of vascular research 2012, 49(3):185-197.

13. Kahe F, Sharfaei S, Pitliya A, Jafarizade M, Seifirad S, Habibi S, Chi G: Coronary artery tortuosity: a narrative review. Coronary artery disease 2020, 31(2):187-192.

14. Zegers ES, Meursing BT, Zegers EB, Oude Ophuis AJ: Coronary tortuosity: a long and winding road. Netherlands heart journal : monthly journal of the Netherlands Society of Cardiology and the Netherlands Heart Foundation 2007, 15(5):191-195.

15. Xie X, Wang Y, Zhu H, Zhou J: Computation of hemodynamics in tortuous left coronary artery: a morphological parametric study. Journal of biomechanical engineering 2014, 136(10):101006.

16. Malve M, Gharib AM, Yazdani SK, Finet G, Martinez MA, Pettigrew R, Ohayon J: Tortuosity of coronary bifurcation as a potential local risk factor for atherosclerosis: CFD steady state study based on in vivo dynamic CT measurements. Annals of biomedical engineering 2015, 43(1):82-93.

17. Zimmermann FM, Omerovic E, Fournier S, Kelbaek H, Johnson NP, Rothenbuhler M, Xaplanteris P, Abdel-Wahab M, Barbato E, Hofsten DE et al: Fractional flow reserve-guided percutaneous coronary intervention vs. medical therapy for patients with stable coronary lesions: meta-analysis of individual patient data. European heart journal 2019, 40(2):180-186.

18. Li Y, Shi Z, Cai Y, Feng Y, Ma G, Shen C, Li Z, Liu N: Impact of coronary tortuosity on coronary pressure: numerical simulation study. PloS one 2012, 7(8):e42558. 


\section{Figure Legend}

Figure 1 Angiography of stenotic coronary artery with coronary artery tortuosity. A. B. Angiography of stenotic coronary artery without coronary tortuosity (CT).

Figure 2 A. Geometry and Pressure contour of the CT model. B. Geometry and Pressure contour of the Non-CT (NCT) model.

Figure 3 A. Pressure profiles in the Pre-stenotic and Post-stenotic domain of the CT model. B. Pressure profiles in the Pre-stenotic and Post-stenotic domain of the NCT model.

Table 1: The pressure difference in the artery zone and FFR calculation under the conditions of CT and NCT.

\begin{tabular}{|c|c|c|c|c|c|c|c|c|}
\hline \multirow{3}{*}{ Outlet } & \multicolumn{2}{|c|}{ The peak } & \multicolumn{2}{|c|}{ The low } & \multirow{2}{*}{\multicolumn{2}{|c|}{ Fluctuation range $(\mathrm{Pa})$}} & \multirow{3}{*}{ FFR } & \multirow{3}{*}{$\operatorname{AS}(\%)$} \\
\hline & \multicolumn{2}{|c|}{ Pressure $(\mathrm{Pa})$} & \multicolumn{2}{|c|}{ pressure $(\mathrm{Pa})$} & & & & \\
\hline & $\mathrm{P}_{\mathrm{a}}$ & $\mathrm{P}_{\mathrm{d}}$ & $\mathrm{P}_{\mathrm{a}}$ & $P_{d}$ & $\mathrm{P}_{\mathrm{a}}(\%)$ & $\mathrm{P}_{\mathrm{d}}(\%)$ & & \\
\hline CT Load & 5521 & 4206 & 2486 & 2296 & $3035(55.0 \%)$ & $1910(45.4 \%)$ & 0.823 & $75 \%$ \\
\hline NCT Load & 3684 & 2366 & 2287 & 2098 & $1399(38.0 \%)$ & $268(7.27 \%)$ & 0.767 & $75 \%$ \\
\hline
\end{tabular}

$\mathrm{FFR}=$ Fractional flow researve $; \mathrm{Pa}=$ Pressure at the aortic stenosis $; \mathrm{Pd}=$ Pressure distal to the coronary stenosis; AS $=$ Area stenosis 


\section{Figures}

A

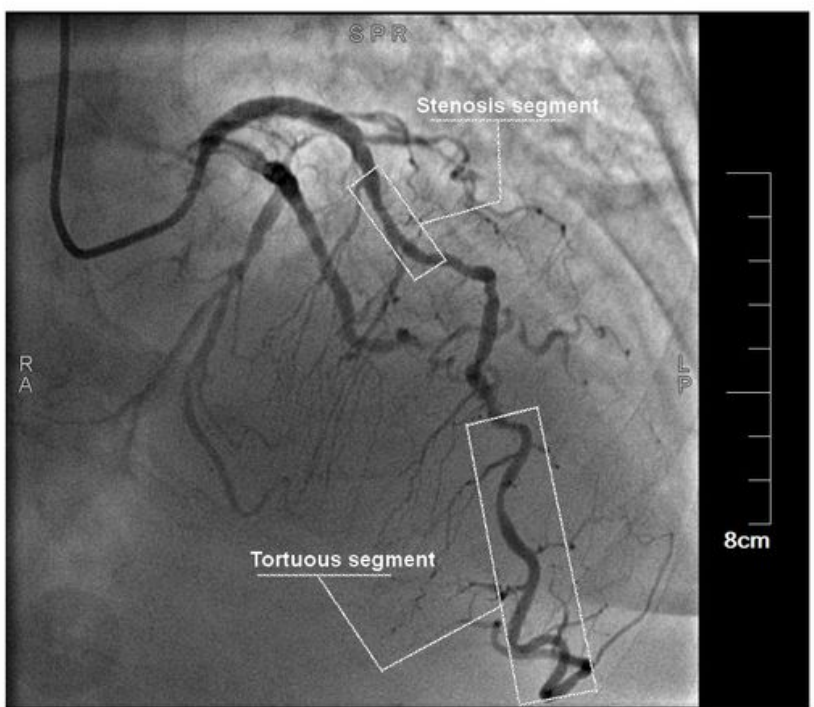

B

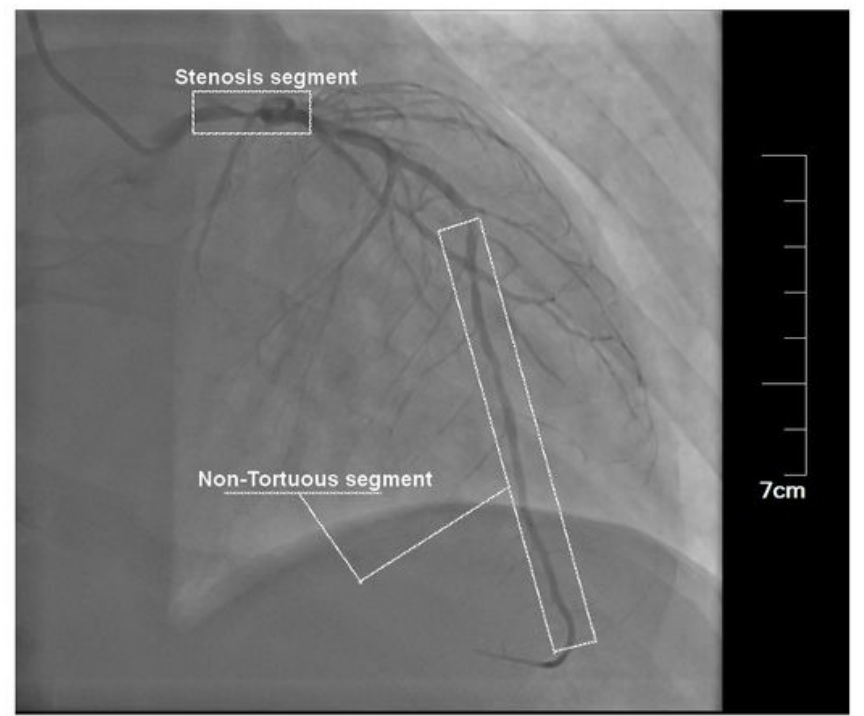

Figure 1

Angiography of stenotic coronary artery with coronary artery tortuosity. A. B. Angiography of stenotic coronary artery without coronary tortuosity (CT).

A

B
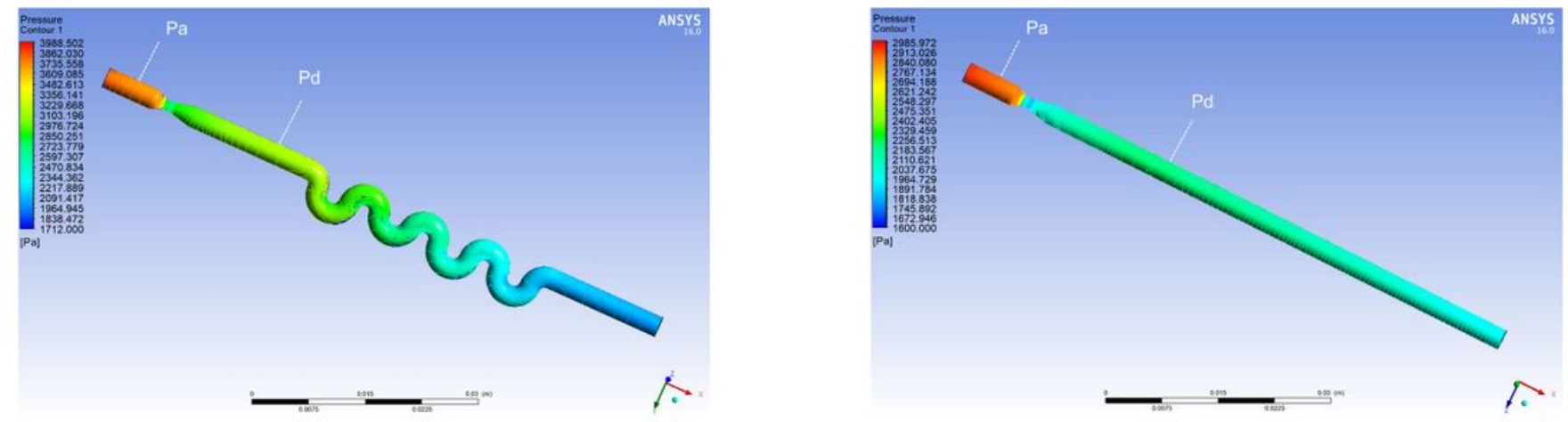

Figure 2

A. Geometry and Pressure contour of the CT model. B. Geometry and Pressure contour of the Non-CT (NCT) model. 

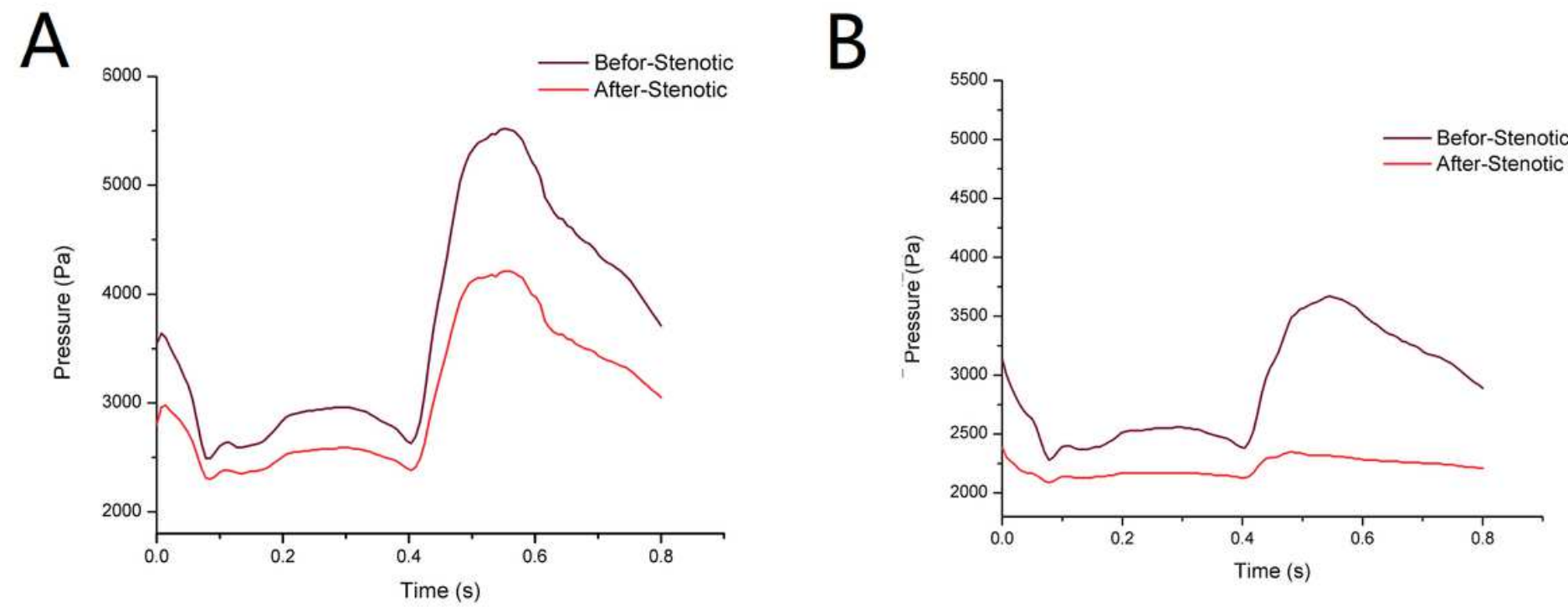

\section{Figure 3}

A. Pressure profiles in the Pre-stenotic and Post-stenotic domain of the CT model. B. Pressure profiles in the Pre-stenotic and Post-stenotic domain of the NCT model. 